\title{
Multifractal concentrations of inertial particles in smooth random flows
}

\author{
By JÉRÉMIE BEC \\ E-mail: jeremie.bec@roma1.infn.it \\ Dipartimento di Fisica, Università La Sapienza, P.zzle Aldo Moro 2, 00185 Roma, Italy. \\ Département Cassiopée, Observatoire de la Côte d'Azur, BP4229, 06304 Nice Cedex 4, France.
}

(16 February 2004)

Collisionless suspensions of inertial particles (finite-size impurities) are studied in twoand three-dimensional spatially smooth flows. Tools borrowed from the study of random dynamical systems are used to identify and to characterise in full generality the mechanisms leading to the formation of strong inhomogeneities in the particle concentration.

Phenomenological arguments are used to show that in two dimensions, the positions of heavy particles form dynamical fractal clusters when their Stokes number (nondimensional viscous friction time) is below some critical value. Numerical simulations provide strong evidence for the presence of this threshold in both two and three dimensions and for particles not only heavier but also lighter than the carrier fluid. In two dimensions, light particles are found to cluster at discrete (time-dependent) positions and velocities in some range of the dynamical parameters (the Stokes number and the mass density ratio between fluid and particles). This regime is absent in the three-dimensional case for which evidence is that the (Hausdorff) fractal dimension of clusters in phase space (position-velocity) remains always above two.

After relaxation of transients, the phase-space density of particles becomes a singular random measure with non-trivial multiscaling properties, whose exponents cannot be predicted by dimensional analysis. Theoretical results about the projection of fractal sets are used to relate the distribution in phase space to the distribution of the particle positions. Multifractality in phase space implies also multiscaling of the spatial distribution of the mass of particles. Two-dimensional simulations, using simple random flows and heavy particles, allow the accurate determination of the scaling exponents: anomalous deviations from self-similar scaling are already observed for Stokes numbers as small as $10^{-4}$.

\section{Introduction}

Suspensions of dust, droplets, bubbles or other kind of small particles in turbulent incompressible flows are present in many stirring and mixing problems encountered in both natural and industrial situations. Such impurities typically have a finite size and a mass density different from that of the carrier fluid. They cannot be described as simple passive tracers, that is point-like particles with negligible mass advected by the fluid; an accurate model for their motion must take into account inertia effects due to the finiteness of their sizes and masses. These inertial particles interact with the fluid through a viscous Stokes drag and thus their motion typically lags behind that of passive tracer particles. The dynamics of the latter is governed by a conservative dynamical system when the carrier flow is incompressible (because volume is conserved), but inertial particles have, 
as we shall see, dissipative dynamics. While an initially uniform distribution of passive tracers remains uniform at any later time, the spatial distribution of inertial particles develops strong inhomogeneities.

Such a phenomenon, frequently referred to as preferential concentration, is associated with the presence of regions with either extremely high or low concentrations. Their characterisation plays an essential role in the understanding of natural and industrial phenomena. Instances are optimisation of combustion processes in the design of Diesel engines (Elperin et al. 2003), the growth of rain drops in subtropical clouds (Pinskv \& Khain 1997; Falkovich et al. 2002), the appearance of planetesimals in the early stage of planet formation in the Solar system (Weidenschilling 1995; Cuzzi et al. 2001), coexistence problems between several species of plankton (Squires \& Yamazaki 1995; Károlvi et al. 2000) and other environmental problems (see, e.g., Seinfeld 1986). For such applications it is recognised that a key problem is the prediction of the collision or reaction rates and their associated typical time scales. The traditional way to estimate the latter goes back to Tavlor (1921) and makes use of diffusion theory. The time scales obtained in such approaches generally exceed by one or several orders of magnitude those observed in experiments or numerical simulations (see, e.g., Sundaram \& Collins 1997). A full understanding of particle clustering and, in particular, of the fine structures appearing in the mass distribution is crucial for identifying and quantifying the mechanisms responsible for the drastic reduction in time scales.

We propose in this paper an original approach leading to a systematic description of inertial particles clustering. This approach is in part inspired by recent major breakthroughs in the study of passive scalar advection by turbulent flows, using Lagrangian techniques (see, e.g., the review of Falkovich et al. 2001). Preferential concentrations are due to the convergence of inertial particle trajectories onto certain sets in the positionvelocity phase space called attractors, which are usually fractals. Of course, these sets are dynamically evolving objects and depend on the history of the carrier flow. When interested only in the spatial distribution of particles, one clearly has to consider the projection on positions of the phase-space attractor. Projection of singular sets are themselves generally singular. As a consequence, an initially uniform distribution of particles will tend to become singular at large times, after relaxation of transients. This is the basic mechanism responsible for the formation of clusters of particles. Use of dissipative dynamical systems tools and, in particular, of methods borrowed from the study of random dynamics, allows a rather complete characterisation of the particle distribution at those scales where the carrier flow can be considered smooth in space. As we shall see, the statistical properties of the spatial distribution of particles in these clusters can be characterised as a function of the dynamical parameters (which depend on the physical properties of the fluid and on the masses and sizes of the particles).

We focus on a simple model which captures most qualitative aspects of inertial particles. In this simplified dynamics, only two effects are included: the Stokes drag proportional to the velocity difference between the particle and the fluid and an added mass term proportional to the acceleration of the fluid volume displaced by the particle. These two terms are associated to two parameters: the Stokes number $S t$ proportional to the cross section of the particle and the mass density ratio between the particle and the surrounding fluid. We identify and characterise the different regimes of the dynamics appearing when varying these two parameters.

The paper is organised as follows. In $\$ 2$ we derive a simple model for the dynamics as an approximation of the full Newton equation describing the motion of particles; in particular, we justify its relevance in some asymptotic range of the physical parameters of the problem. In 3 this model is used to relate the local dynamics of the particles 
to the local properties of the carrier flow; this permits to perform a complete stability analysis of the particle dynamics. In 4 after a brief introduction to the tools used in studies of dissipative dynamics with particular emphasis on Lyapunov exponents (4.1), phenomenological arguments are used to extend the local approach to the global dynamics in order to show that below a critical value of the Stokes number the particles concentrate on fractal dynamical clusters in the position space (4.2); this is confirmed by numerical experiments presented in 4.3 A more precise description of the statistical properties of these clusters is performed in $\$ 5$ where it is shown that the distribution of particle actually has multifractal properties, even at very small Stokes numbers. Finally, 6 encompasses concluding remarks and open questions.

\section{Dynamical model for the particles}

We consider very diluted suspensions of inertial particles where collisions, particle-toparticle hydrodynamical interactions and retroaction of the particles on the motion of the fluid can be ignored. It is assumed that the carrier flow has moderate Reynolds numbers and that the particle radius is smaller than their dissipation scale. Under the additional assumption that the Reynolds number based on the particle size and its relative velocity with respect to the fluid is sufficiently small, Maxev \& Rilev (1983) proposed approaching the flow surrounding the small sphere by a Stokes flow. Neglecting both the effects of gravity and the Faxén corrections, the motion $\mathbf{X}(t)$ of an inertial particle is then solution to the following Newton equation

$$
\begin{aligned}
m_{p} \ddot{\mathbf{X}}= & m_{f} \frac{\mathrm{D} \boldsymbol{u}}{\mathrm{D} t}(\mathbf{X}, t)-6 \pi a \mu[\dot{\mathbf{X}}-\boldsymbol{u}(\mathbf{X}, t)]-\frac{m_{f}}{2}\left[\ddot{\mathbf{X}}-\frac{\mathrm{d}}{\mathrm{d} t}(\boldsymbol{u}(\mathbf{X}, t))\right] \\
& -\frac{6 \pi a^{2} \mu}{\sqrt{\pi \nu}} \int_{0}^{t} \frac{\mathrm{d} s}{\sqrt{t-s}} \frac{\mathrm{d}}{\mathrm{d} s}[\dot{\mathbf{X}}-\boldsymbol{u}(\mathbf{X}, s)] .
\end{aligned}
$$

The dots denote time derivatives, $\boldsymbol{u}$ is the velocity field of the carrier fluid, D $\boldsymbol{u} / \mathrm{D} t$ is its derivative along the path of a fluid element, $m_{p}$ is the mass of the particle, $m_{f}$ is the mass of fluid displaced by the particle, $a$ is the radius of the particle and, finally, $\mu$ and $\nu$ are respectively the dynamic and the kinematic viscosities of the fluid. The different forces exerted on the particle are, in order of appearance on the right-hand side of (2.1), the force exerted by the undisturbed flow, the Stokes viscous drag, the added mass effect and the Basset-Boussinesq history force.

For rescaling purposes it is more convenient to write the equation satisfied by the velocity difference between the particle and the carrier fluid $\boldsymbol{w}(t) \equiv \dot{\mathbf{X}}-\boldsymbol{u}(\mathbf{X}, t)$. Indeed, the dynamics involves two velocity scales which are determined by $\boldsymbol{u}$ and $\boldsymbol{w}$ and there is a priori no reason why they should be of the same order of magnitude. Rescaling of space, time, velocity of the carrier fluid and velocity difference by the factors $L, L / U, U$ and $W$, respectively, leads to

$$
\frac{\mathrm{d} \boldsymbol{w}}{\mathrm{d} t}=\frac{\beta-1}{\alpha} \frac{\mathrm{d}}{\mathrm{d} t}(\boldsymbol{u}(\mathbf{X}, t))-\beta(\boldsymbol{w} \cdot \nabla) \boldsymbol{u}(\mathbf{X}, t)-\frac{1}{S t} \boldsymbol{w}-\sqrt{\frac{3 \beta}{\pi S t}} \int_{0}^{t} \frac{\mathrm{d} s}{\sqrt{t-s}} \frac{\mathrm{d} \boldsymbol{w}}{\mathrm{d} s} .
$$

The dynamics depends here on three parameters : the velocity ratio $\alpha \equiv W / U$, the addedmass factor $\beta \equiv 3 m_{f} /\left(m_{f}+2 m_{p}\right)$ and the Stokes number associated to the particle $S t \equiv\left(a^{2} U\right) /(3 \beta \nu L)$. The latter can be also written as $S t=\operatorname{Re}(a / L)^{2} /(3 \beta)$ where $R e \equiv$ $U L / \nu$ is the Reynolds number of the carrier flow. The particle radius $a$ is taken to be smaller than the typical (integral) scale $L$ of the carrier flow. As a consequence, if the Reynolds number is not very large and the particles are not very heavy we must assume 
that the Stokes number is much smaller than unity. Moreover, the requirement of small local Reynolds number in the neighbourhood of the particle - required in Maxey \& Riley derivation of (2.1) - reads here $W a / \nu \ll 1$. It restricts the range of admissible parameters to satisfying $\alpha \ll(\beta S t)^{-1}$. When both $\beta$ and $S t$ are taken order unity, the velocity ratio $\alpha$ must be small compared to unity, meaning that the inertial-particle dynamics must be very close to that of ordinary fluid particles. This can only be achieved by requiring low values of the Stokes number. Hence, in the Maxey \& Riley approach, $\beta$ and $S t$ cannot be simultaneously order unity.

We nevertheless consider here particles whose motion is described by a simplified dynamics where both the second and the last term of (2.2) are neglected. This is clearly the case when the typical velocity difference $\alpha$, and thus the Stokes number are both very small. Such simplified dynamics is also relevant when the particles are much heavier than the fluid $(\beta \ll 1)$, a case of importance for applications involving suspensions of liquid droplets or dust particles in a gas. Under one of these two hypotheses and by introducing what we call here the covelocity $\mathbf{V} \equiv \dot{\mathbf{X}}-\beta \boldsymbol{u}(\mathbf{X}, t)$, it is easy to see that the equation of motion reduces to the $(2 \times d)$-dimensional system

$$
\begin{aligned}
& \dot{\mathbf{X}}=\beta \boldsymbol{u}(\mathbf{X}, t)+\mathbf{V}, \\
& \dot{\mathbf{V}}=\frac{1}{S t}[(1-\beta) \boldsymbol{u}(\mathbf{X}, t)-\mathbf{V}],
\end{aligned}
$$

combined with the initial conditions $\mathbf{X}(0)=\boldsymbol{x}_{0}$ and $\mathbf{V}(0)=(1-\beta) \boldsymbol{u}\left(\boldsymbol{x}_{0}, 0\right)+\alpha \boldsymbol{w}_{0}$. Here $\boldsymbol{w}_{0}$ denotes the non-dimensional initial velocity difference between the particle and the flow. The dynamics described by (2.3) are clearly dissipative. Indeed, the divergence of the right-hand side of (2.3) with respect to the phase-space variables $(\boldsymbol{x}, \boldsymbol{v})$ reduces in the case of divergence-free carrier flow to $-d / S t$, which is negative. This implies that all volumes in position-covelocity phase space are uniformly contracted during time evolution.

\section{Local analysis of the dynamics}

As a first step in describing the mechanisms responsible of particle clustering, we study the time evolution of the position-covelocity phase-space separation $\mathbf{R} \equiv(\delta \mathbf{X}, \delta \mathbf{V})$ between two infinitesimally close trajectories. Since the sizes of inertial particles are within the dissipation range of the carrier flow, the velocity field $\boldsymbol{u}$ can be considered spatially smooth. Velocity gradients are then bounded and velocity differences between two neighbouring locations are linearly proportional to their separation. The separation $\mathbf{R}(t)$ thus obeys an equation obtained by linearising (2.3), namely

$$
\dot{\mathbf{R}}=\mathcal{M}_{t} \mathbf{R}, \quad \mathcal{M}_{t} \equiv\left[\begin{array}{cc}
\beta \boldsymbol{\sigma}(t) & \mathcal{I}_{d} \\
\frac{1-\beta}{S t} \boldsymbol{\sigma}(t) & -\frac{1}{S t} \mathcal{I}_{d}
\end{array}\right] .
$$

Here $\boldsymbol{\sigma}$ denotes the strain matrix of the carrier flow along the trajectory of a particle: $\sigma_{i j}(t) \equiv \partial_{j} u_{i}(\boldsymbol{X}(t), t)$, and $\mathcal{I}_{d}$ stands for the $d$-dimensional identity matrix. Qualitative insight into the local dynamics of the particles is provided by making the stability analysis of (3.1). The eigenvalues of the evolution matrix $\mathcal{M}_{t}$ depend on the local structure of the carrier flow, as we now show. It is easily checked that $\mathcal{M}_{t}$ is a solution to the second-order equation

$$
\mathcal{M}_{t}^{2}+\left(\frac{1}{S t} \mathcal{I}_{2 d}-\beta \Sigma_{t}\right) \mathcal{M}_{t}-\frac{1}{S t} \Sigma_{t}=0_{2 d}
$$



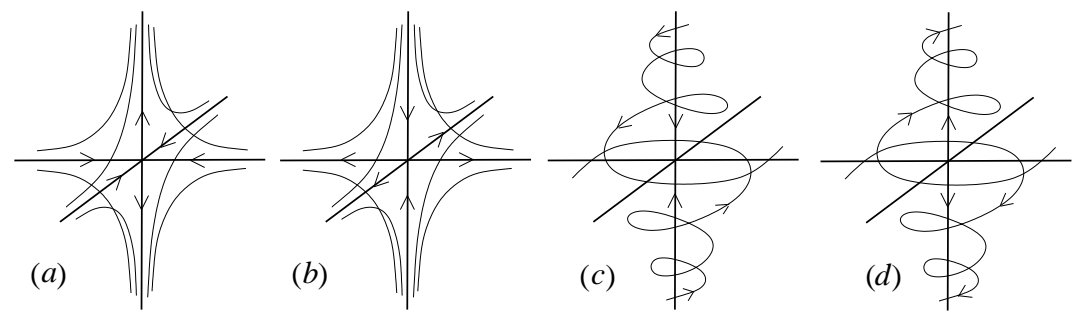

FigURE 1. Local structure of the carrier flow in three dimensions ; four cases are distinguished accordingly to the nature of the three eigenvalues of the strain matrix: if all three are real (hyperbolic case), either two of them are negative and one is positive $(a)$ or two of them are positive and the third is negative $(b)$. When two eigenvalues are complex conjugate (elliptic case), the third one is real and can be either negative $(c)$ or positive $(d)$.

where $\Sigma_{t}$ is the $2 d \times 2 d$ block-diagonal matrix given by

$$
\Sigma_{t} \equiv\left[\begin{array}{lr}
\boldsymbol{\sigma}(t) & 0_{d} \\
0_{d} & \boldsymbol{\sigma}(t)
\end{array}\right]
$$

and $0_{d}$ is the $d$-dimensional square matrix with all entries zero. A straightforward consequence is that, to a given eigenvalue $\gamma$ of the strain matrix, correspond two eigenvalues of the evolution matrix $\mathcal{M}_{t}$ solutions of the quadratic equation

$$
x^{2}+\left(\frac{1}{S t}-\beta \gamma\right) x-\frac{\gamma}{S t}=0 .
$$

The stability analysis of the evolution matrix requires to express the sign of the real part of the solutions to this second-order equation as a function of $\gamma$, and thus depends on the local structure of the carrier flow.

Let us examine the consequence, first in two dimensions. Depending on the sign of the Okubo-Weiss parameter $Q$ (Okubo 1970; Weiss 1991) defined as the determinant of the strain matrix $\sigma$ and thus satisfying $\gamma^{2}=Q$, the incompressible flow of the carrier fluid separates the space in regions of two kinds: the hyperbolic regions where $Q>0$ and where the strain matrix $\boldsymbol{\sigma}$ has two opposite real eigenvalues and the elliptic regions where $Q<0$, so that the two eigenvalues are imaginary numbers. A simple analysis of (3.4) shows that in the hyperbolic regions, the evolution matrix $\mathcal{M}_{t}$ of the particle dynamics has three stable and one unstable eigendirections in the four-dimensional position-covelocity phase space. In the elliptic regions, one must distinguish two cases: if $\beta<1$ (particles heavier than the fluid), there are two stable and two unstable eigendirections, whereas if $\beta>1$ (lighter particles), there are always four stable directions. This local analysis suggests that in two dimensions, heavy particles are excluded from the vortices (elliptic regions) and tend to concentrate within the filaments (hyperbolic regions) of the carrier flow, whereas light particles tend to cluster in the vortices.

In three dimensions, there are four possible local structures of the incompressible carrier flow represented schematically in figure 1 They can also be divided into elliptic and hyperbolic cases depending on the presence or not of complex eigenvalues.

- Hyperbolic regions $(a, b)$ : the local dynamics of the particles has five stable and one unstable eigendirections in the case $(a)$ and four stable and two unstable in the case $(b)$;

- Elliptic regions $(c, d)$ : there is generally at least one unstable eigendirection; the exception is case $(c)$ for light particles $(\beta>1)$; all six eigendirections are then stable provided the real negative eigenvalue $\gamma$ of the strain matrix satisfies $\gamma<-S t / \beta$.

The three-dimensional situation looks hence similar to the two-dimensional one: the local analysis suggests that particles lighter than the carrier fluid concentrate in the 
rotation regions while heavier particles tend to be ejected from them and concentrate in the strain regions. The model is hence able to catch a qualitative property of inertial particles dynamics which plays a central role in the phenomenological understanding of their preferential concentration. As stated for instance in Squires \& Eaton (1991), the appearance of inhomogeneities in the distribution of particles is generally explained by the presence of persistent structures in the carrier flow. The latter are responsible for a deterministic motion of the particles during which their inertia affects their relative motion with respect to the carrier flow: phenomenology indicates that heavy particles are ejected from eddies while light particles tend to concentrate in their cores. This is indeed what our local analysis predicts. However we shall see in the next section that, although preferential concentrations of inertial particles are triggered by the local mechanisms just discussed, it is the dissipative character of the dynamics which is solely responsible for the eventual strong clustering of particles.

\section{Threshold in Stokes number for the formation of fractal clusters}

\subsection{Dissipative dynamics and Lyapunov exponents}

The temporal evolution of the separation $\mathbf{R}(t)$ between two infinitesimally close trajectories of the phase space can be expressed in terms of the Green function $\mathcal{J}_{t}$ associated to the linearised dynamics (3.1). We indeed have

$$
\mathbf{R}(t)=\mathcal{J}_{t} \mathbf{R}(0), \quad \mathcal{J}_{t} \equiv \mathcal{T} \exp \int_{0}^{t} \mathrm{~d} \tau \mathcal{M}_{\tau},
$$

where $\mathcal{T} \exp$ denotes the time-ordered exponential of matrices and $\mathcal{M}_{t}$ is the evolution matrix defined in (3.1). The symmetrical matrix $\mathcal{J}_{t}^{\top} \mathcal{J}_{t}$ has positive eigenvalues that can be written $\mathrm{e}^{2 \mu_{1}(t) t}, \ldots, \mathrm{e}^{2 \mu_{2 d}(t) t}$. The $\mu_{j}$ 's are called the stretching rates (or the finite-time Lyapunov exponents) and are generally labelled in non-increasing order $\mu_{1}(t) \geqslant \cdots \geqslant \mu_{2 d}(t)$. They measure the time-evolution of infinitesimal volumes of phase space. $\mu_{1}$ measures the exponential growth rate of the distance between two neighbouring trajectories, $\mu_{1}+\mu_{2}$ measures that of areas defined by three trajectories, etc. The sum of the $2 d$ stretching rates controls the time evolution of phase-space $2 d$-dimensional volumes and is expressible in terms of the trace of the Green matrix $\mathcal{J}_{t}$. It is easily shown that $\mu_{1}+\cdots+\mu_{2 d}=-d /$ St.

The long-time behaviour of the local dynamics is dominated by the almost-sure convergence of the stretching rates to the classical Lyapunov exponents $\lambda_{j}=\lim _{t \rightarrow \infty} \mu_{j}(t)$. The multiplicative ergodic theorem (Oseledets 1968) ensures that, under some ergodicity hypothesis on the dynamics, the Lyapunov exponents are independent of both the realisation of the random carrier flow and of the peculiar trajectory $\mathbf{X}(t)$ around which the linearised dynamics is considered. The Lyapunov exponents are linked to many fundamental features of the dynamics. For instance, when the largest Lyapunov exponent $\lambda_{1}$ is negative, the stability of the linearised system is ensured and all the particle trajectories are converging together, thereby leading to a somewhat degenerate statistical steady state in which all the mass is concentrated in discrete time-dependent points in phase space. However when $\lambda_{1}$ is positive, the situation is more complex: the dynamics is then said to be chaotic and stability is ensured only if the initial phase-space separation $\mathbf{R}(0)$ is orthogonal to the subspace generated by the eigendirections associated to the positive Lyapunov exponents.

When $\lambda_{1}>0$ the long-time dynamics has richer features, characterised by the convergence of the particle trajectories to complex dynamical structures called attractors. These 


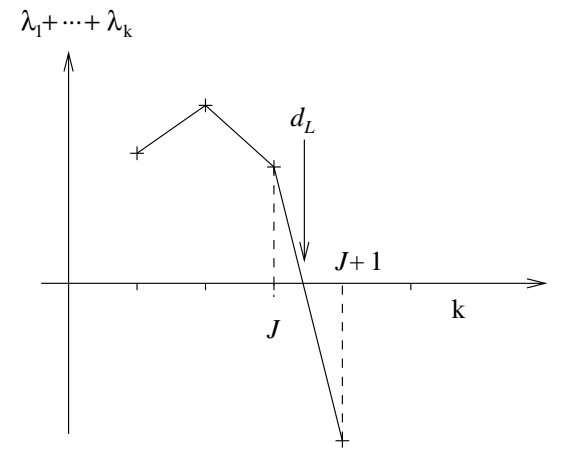

Figure 2. The Lyapunov dimension $d_{L}$ is defined by interpolating to non-integer values the exponential growth rate of phase-space $k$-dimensional volumes obtained from the sum of the $k$ largest Lyapunov exponents.

are generally (evolving) fractal sets of the phase space and can be characterised by their Hausdorff dimension $d_{H}$, which is expected to coincide with the box-counting dimension in non-degenerate cases. The appearance of attractors can be seen as the result of a competition between stretching and folding effects that occur during the chaotic motion of the particles. As a consequence, the properties of these attractors, and in particular their dimension, depend on the stretching rates of the dynamics. The positive $\mu_{j}$ 's are responsible for stretching in their associated eigendirections, while the negative rates give contraction and hence lead to folding. Following this kind of approach, Kaplan \& Yorke (1979) proposed to estimate the dimension of the attractor by the Lyapunov dimension, defined as

$$
d_{L} \equiv J-\frac{\lambda_{1}+\cdots+\lambda_{J}}{\lambda_{J+1}}, \quad \text { where } \quad \lambda_{1}+\cdots+\lambda_{J} \geqslant 0 \quad \text { and } \quad \lambda_{1}+\cdots+\lambda_{J+1}<0 .
$$

This non-random number can be interpreted heuristically as the dimension of phasespace objects that keep a constant volume during time evolution. As already stated above, the long-time evolution of $k$-dimensional volumes is governed by the sum of the $k$ first Lyapunov exponents. The Lyapunov dimension is obtained by interpolating this sum to non-integer values and determining where it vanishes (see figure 2). It was shown by Douadv \& Oesterle (1980) that it serves as a rigorous upper-bound for the Hausdorff dimension $d_{H}$; this will be used in next subsection to show that below a critical value of the Stokes number, the particles form fractal clusters in the position space. As we shall see in 95 the Lyapunov dimension is actually equal to the information dimension associated to the steady-state phase-space density of particles and is thus related to the small-scale properties of the mass distribution of inertial particles.

\subsection{A heuristic approach in two dimensions}

We now give a heuristic argument which predicts the presence of a threshold in Stokes number for clustering of heavy particles. This result, already described briefly in Bed (2003), is here presented in more details.

As already stated, the particles concentrate on dynamical attractors in the positioncovelocity phase space. The main idea of the argument presented here comes from a trivial observation. Focusing on positions while ignoring covelocities amounts to projecting the attractor from the $2 d$-dimensional phase space onto the $d$-dimensional space of particle positions. A standard result on the geometry of fractal sets (see, e.g., Falconer 1986) is that, when the Hausdorff dimension $d_{H}$ of the attractor is less than the dimension of 
the projection space (here, $d$ ), the projection of the fractal is itself a fractal set with Hausdorff dimension $d_{H}$. If however $d_{H}>d$, the projection has dimension $d$. Now, using the Lyapunov dimension $d_{L}$ as a rigorous upper bound of the fractal dimension $d_{H}$ of the phase-space attractor, the condition $d_{L}<d$ is a sufficient condition for particles to form fractal clusters in position space. The next step is to find a condition ensuring that the Lyapunov dimension is less than the space dimension. From its definition (4.2), it is clear that $d_{L}<d$ if the sum of the $d$ largest Lyapunov exponents is negative. Hence, the problem reduces to finding a sufficient condition for $\lambda_{1}+\cdots+\lambda_{d}<0$.

To estimate the $d$ largest Lyapunov exponents we first consider the stability exponents defined as

$$
\mu_{j} \equiv \lim _{t \rightarrow \infty} \frac{1}{t} \ln \alpha_{j}(t)
$$

where the $\alpha_{j}(t)$ 's are the eigenvalues of the Jacobi matrix $\mathcal{J}_{t}$ defined in (4.1), which are usually complex numbers. Although there is no equivalent of the Oseledets ergodic theorem for such complex exponents, it is usually supposed that the limit in (4.3) exists in generic situations and depends neither on the realisation of the carrier velocity field, nor on the particular trajectory along which they are calculated (see, e.g. Goldhirsch et al. 1987). The real parts of the stability exponents generally differ from the Lyapunov exponents. It is however possible to obtain inequalities between these two sets of exponents, using Browne's theorem (see, e.g., for details Marcus \& Mind 1992). We will also need the strain exponents $\eta_{\ell}$ analogous to the $\mu_{j}$ 's but this time associated to the $(d \times d)$ dimensional matrix $\mathcal{T} \exp \int_{0}^{t} \mathrm{~d} \tau \boldsymbol{\sigma}(\tau)$.

We now focus on the case of heavy particles $(\beta<1)$ embedded in a two-dimensional incompressible carrier flow. Note first that, as we have seen from the local analysis in the previous section, the heavy particles tend to concentrate in the filamentous structures of the flow (hyperbolic regions). The eigenvalues associated to the local dynamics of the particles are given by the quadratic relation (3.4) in terms of those associated to the strain matrix of the carrier flow. Now comes the heuristic point. Let us assume that the same quadratic relation allows us to express, at least in an approximate way, the stability exponents $\alpha_{j}$ 's as a function of the strain exponents $\eta_{\ell}$ 's defined abovet. The latter are expected to be real since they are dominated by the hyperbolic regions of the flow. In two dimensions there are only two strain exponents $+\eta$ and $-\eta$. Under these hypotheses a sufficient condition for fractal clustering of the particles can be written

$$
\text { St } \leqslant \frac{1}{\beta^{2} \eta}\left(\beta-2+2 \sqrt{1-\beta+\beta^{2}}\right) .
$$

Observe that the (non-dimensional) strain exponent $\eta$ is calculated along the path of inertial particles and not along that of fluid particles. As the heavy particles spend a long time in the hyperbolic regions, the strain should satisfy $\eta \geqslant \lambda_{f} L / U$, where $\lambda_{f}$ denotes the positive Lyapunov exponents associated to the dynamics of fluid particles. Using this relation in (4.4) we obtain that there is clustering if

$$
S t \leqslant \frac{U}{\beta^{2} \lambda_{f} L}\left(\beta-2+2 \sqrt{1-\beta+\beta^{2}}\right) .
$$

Note that this sufficient condition gives heuristic evidence that clustering already occurs at small Stokes numbers, although the Stokes drag is then very strong, and thus the inertial particle motion is very close to that of simple passive tracers. This is consistent

$\dagger$ This assumption would be exact if one could replace time-ordered exponentials appearing in the definition of the various Green functions by ordinary exponentials; of course, this is not the case here. 
with predictions made by Balkovskv et al. (2001). Their approach is based on the observation of Maxev (1987) that the discrepancy between inertial particle dynamics and passive tracers occuring when $S t \ll 1$ can be captured by a spatial Taylor expansion of the particle velocity field, leading to a model in which the particle is advected by a synthetic flow comprising a small compressible component. Such an expansion allowed Balkovsky et al. to show that moments of particle density grow exponentially in times, which is indeed indicative of the formation of clusters.

The existence of a threshold in Stokes number for particle clustering requires that for large-enough values there be no clustering. It is easily checked from equations (2.3) governing inertial-particle dynamics that, when increasing the Stokes number, the particle are less and less influenced by the carrier flow. More precisely, in the limit $S t \rightarrow \infty$, their motion becomes purely ballistic and their distribution in position space tends to become uniform, ensuring absence of fractal clusters for large-enough Stokes number.

Note that the same type of arguments when applied to light particles (with $\beta>1$ ) whose dynamics is dominated by elliptic regions of the flow, would predict that they always concentrate on points located in the cores of vortices (clusters of dimension $d_{H}=$ $0)$. As we shall see from the numerical experiments of the next subsection, there are ranges of values of the parameters $\beta$ and $S t$ for which this strong clustering occurs in two dimensions, but for other values the light particles can also form either fractal clusters or fill the whole space. This indicates the limits of our heuristic approach. The arguments used in the case of heavy particles do not apply to light particles in the elliptic regions since the eigenvalues of the local dynamics are then complex and cannot be used to estimate the stability exponents. Moreover, in order to match the limit $S t \rightarrow 0$ in which the motion of light particles recovers that of fluid particles, it is clear that their dynamics is influenced by their stay in the hyperbolic regions, even if this stay is shortened by inertial effects. The phenomenological derivation of the sufficient condition (4.5) was just meant to give an idea of the physical mechanisms responsible for particles clustering. Its qualitative validity is however confirmed numerically, as we shall now see.

\subsection{Numerical evidence for fractal clustering in two and three dimensions}

In our simulations the inertial particles positions are confined to a periodic domain of size $L$. The prescribed carrier flow is a solution to the forced Stokes equation

$$
\partial_{t} \boldsymbol{u}=\nu \Delta \boldsymbol{u}+\boldsymbol{f}(\boldsymbol{x}, t), \quad \nabla \cdot \boldsymbol{u}=0,
$$

where $\boldsymbol{f}(\boldsymbol{x}, t)$ is a space-periodic, isotropic and homogeneous Gaussian random forcing concentrated at spatial scales of the order of the box size $L$ and delta-correlated in time. Such a carrier flow provides control over the statistical properties of the Gaussian velocity field $\boldsymbol{u}$ and allows accurate numerics. In practice we take $\nu=1$ and assume that the velocity field has the lowest reasonable number of spatial Fourier modes for which isotropy is approximately ensured: 9 in two dimensions and 27 in three dimensionst. Note that two-dimensional flows satisfying (4.6) were also considered by Sigurgeirsson \& Stuart (2002) who proved in this setting existence of the random dynamical attractor for very heavy particles.

The individual trajectories of inertial particles are integrated using a fourth-order Runge-Kutta scheme. Choosing a carrier fluid velocity with rather few Fourier modes makes it unnecessary to interpolate the velocity at particle locations, since we can calculate it by direct summation of the Fourier series. Fully resolving the finest structures of

$\dagger$ Fewer Fourier modes may actually lead to degenerate situations and in particular to insufficiently mixing dynamics. 


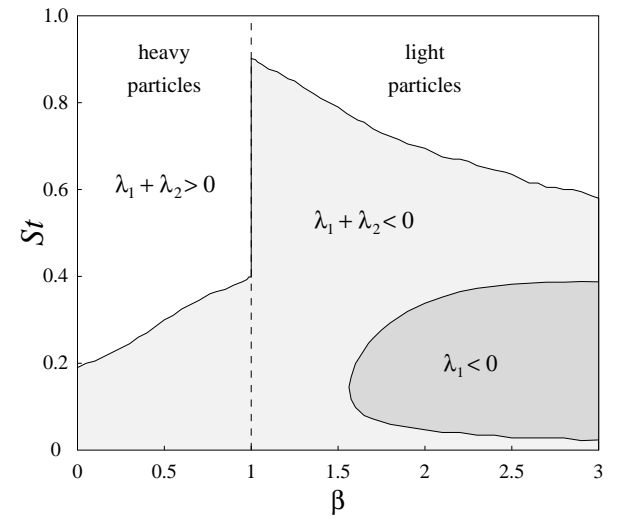

(a) $d=2$

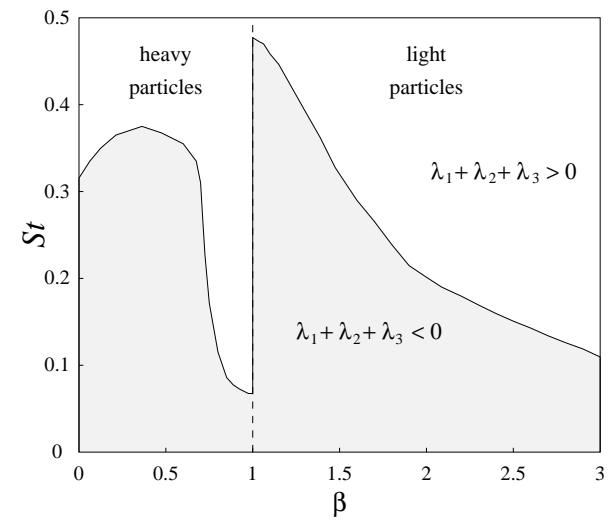

(b) $d=3$

Figure 3. Phase diagrams in the parameter space $(\beta, S t)$ for $d=2(a)$ and $d=3(b)$ representing the different regimes of the dynamics corresponding to different behaviours of the Lyapunov exponents. For parameter values in the white area (such that $\lambda_{1}+\cdots+\lambda_{d}>0$ ), the distribution of particle fills the whole position space. In the light-gray area the Lyapunov dimension is less than $d$ (i.e. $\lambda_{1}+\cdots+\lambda_{d}<0$ ) and particles concentrate on fractal clusters. The darker gray area which is only present in the two-dimensional case corresponds to the regime for which all Lyapunov exponents are negative: the asymptotic distribution of particles is then atomic.

the spatial particle distribution requires a strict control over the small-scale dynamics, as is here the case.

We present in this section measurements of the Lyapunov exponents associated to the dynamics of the particles. To estimate these quantities, we use the standard method developed by Benettin et al. (1980) - see also the book by Crisanti et al. (1993) for a precise description of this method. The idea is to integrate the time evolution of $2 d$ infinitesimal separations $\boldsymbol{R}_{1}, \ldots, \boldsymbol{R}_{2 d}$ governed by the linearised dynamics (3.1) and along the path of a given particle. The Lyapunov exponents are obtained from the exponential growth rates of the distances, surfaces, volumes, etc. defined by these infinitesimal vectors. In order to prevent numerical errors from accumulating and the length of the $\boldsymbol{R}_{j}$ 's from increasing too rapidly, their time evolution must be accompanied by frequent renormalisation, $\dagger$ using, for instance, the Gram-Schmidt procedure. In practice, to obtain the Lyapunov exponents for given values of the parameters $\beta$ and $S t$, we integrate the linearised system for $10^{5}$ turn-over times performing a renormalisation of the vectors $\boldsymbol{R}_{1}, \ldots, \boldsymbol{R}_{2 d}$ every tenth of turn-over time.

Figure $3(a)$ shows the phase diagram obtained numerically in the two-dimensional case, which divides the parameter space $(\beta, S t)$ into three different regimes. The lightgray area represents those values for which the sum of the two first Lyapunov exponents is negative. The Lyapunov dimension is there smaller than the dimension $d=2$ of the position space and the particles form fractal clusters. The second regime appearing in the dynamics is when the sum of the two first Lyapunov exponents is positive (white area). This corresponds to the case when the Lyapunov dimension is larger than two and the particles fill the whole domain. The last regime, represented by the dark-gray area in figure $3(a)$, corresponds to values of the dynamical parameters for which the largest Lyapunov exponent $\lambda_{1}$ is negative. This strong clustering happens only when the particles are lighter than the fluid. They form there point-like clusters and the associated mass

$\dagger$ Here the term "renormalisation" is to be taken literally, meaning just "making the norm finite". 
distribution is said to be atomic. In the case of bounded domains (such as considered here) it corresponds to the large-time convergence of all particle trajectories towards a single trajectory.

In three dimensions, the situation is rather different. As shown in figure $3(b)$, there are only two different possible regimes in the particle dynamics: one corresponding to a Lyapunov dimension larger then the position-space dimension and in which particles fill the whole space; the other one associated to a Lyapunov dimension smaller than $d$ and where particles concentrate onto fractal clusters. Surprisingly, there is no evidence for the presence of a region in the parameter plane where the particles cluster on a set of dimension less than two. A systematic investigation for a large number of different values of the parameters $\beta$ and $S t$ was done. In particular, the Lyapunov exponents were calculated for forty different values of $S t$ at $\beta=2.9$ for which clustering would have been expected. Results indicate that the sum of the two largest Lyapunov exponents always remains positive. This observation contradicts the phenomenological understanding of clustering which predicts that light particles may form clusters of dimension between one and two in the core of the vortices. The absence of such a behaviour is not due to the choice of the model, otherwise point-like clusters would also be absent in two dimensions. A possible explanation is related to the properties of the carrier flow considered here. It could be that in our setting, the velocity field $\boldsymbol{u}$ does not present rotational structures which are strong enough and sufficiently persistent to permit such a clustering of the particles. On the one hand, we have seen from the local analysis of 3 that the elliptic regions of the carrier flow are stable for light particles only if the Stokes number is sufficiently small. On the other hand, St has to be sufficiently large for the particles to have enough inertia to separate from the fluid motion. It may happen that these two conditions are here incompatible. It would be of interest to confirm the absence of pointlike clusters by systematic investigations in different flow fields (and in particular realistic ones); this will be done in future work.

The separatrix between the regimes associated to different signs of the sum of the $d$ largest Lyapunov exponents has a discontinuity at $\beta=1$ in both two and three dimensions. This singular behaviour is due to the degenerate dynamics associated to particles with the same density as the fluid (such particles are usually referred to as neutrally buoyant particles). It is indeed clear from (2.3) that when $\beta=1$, the covelocity does not depend anymore on the velocity of the carrier flow; it simply relaxes exponentially from its initial value to zero. As a consequence, the subspace of covelocities is an eigenspace for the linearised dynamics associated to a Lyapunov exponent equal to $-1 / S t$ and with multiplicity $d$. Incompressibility of the carrier flow then implies that $\lambda_{1}+\cdots+\lambda_{d}=0$ on the whole line $\beta=1$ of the parameter space, which partly explains the singular behaviour observed here.

More quantitative information is provided by the behaviour of the Lyapunov dimension $d_{L}$ as a function of the Stokes number. Figure 4 shows the results obtained from both two- and three-dimensional simulations in the case of very heavy particles $(\beta=0)$. First, we observe that the dimension of the attractor indeed tends to the space dimension $d$ at low Stokes numbers. More precisely, in both two and three dimensions, the Lyapunov dimension behaves in this asymptotic regime as $d_{L} \simeq d-C S t^{2}$. Such a quadratic behaviour near vanishing Stokes numbers has been obtained theoretically by Balkovskv et al. (2001) using the method of advection by a synthetic compressible flow, already mentioned. At large Stokes numbers, the Lyapunov dimension becomes larger and larger, eventually reaching $2 d$ when $S t \rightarrow \infty$. Between these two asymptotic regimes, there is a range of Stokes numbers for which the Lyapunov dimension - and thus the fractal dimension of the attractor - is smaller than $d$. For such Stokes numbers, the particles form fractal clusters. 


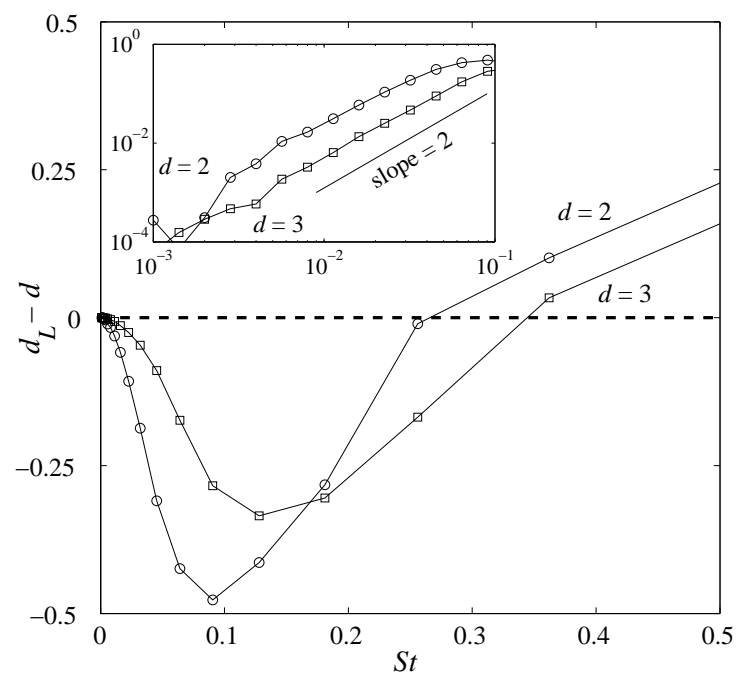

Figure 4. Difference $d_{L}-d$ between the Lyapunov dimension associated to the dynamics of very heavy particles $(\beta=0)$ and the position-space dimension, as a function of the Stokes number $S t$ (circles: $d=2$, squares: $d=3$ ). The critical Stokes number corresponds to the value for which $d_{L}=d$. Upper-left inset : same in log-log coordinates showing evidence for a quadratic behaviour at low Stokes numbers.

The graphs of the Lyapunov dimension against the Stokes number, shown in figure 4 display a minimum around $S t=0.09$ for $d=2$ and $S t=0.14$ for $d=3$. These are the Stokes numbers for which the strongest clustering takes place. Finally, these experiments also confirm the existence of a critical value for the Stokes number below which fractal clustering occurs; on the figure it corresponds to the value for which the curves cross the line $d=d_{L}$ (around $S t=0.24$ for $d=2$ and $S t=0.32$ for $d=3$ ).

Qualitative insight into clustering is provided by figure 5 where snapshots of the position of $10^{4}$ very heavy particles illustrate the different regimes arising in the twodimensional dynamics when increasing the Stokes number. First, when the Stokes number is very small (here $S t=10^{-3}$ for figure [5] $a$ ), the particles are rather densely distributed but it is possible to observe fractal clusters with a dimension very close to the space dimension $d$. This picture illustrates the highly singular nature of the vanishing Stokes number limit. Note also that, as the Stokes number becomes very small, the dynamical fractal clusters are becoming denser and more space-filling. Conversely, as we increase $S t$, the dimension of the clusters decreases and large empty areas appear (figure $\mathbf{5} b$ ). This process continues up to the value of $S t$ corresponding to the strongest clustering. Above this value, the dimension of clusters increases as a function of $S t$ and caustic-like structures become conspicuous in the particle spatial distribution (figure 5 ). Finally, when the Stokes number is larger than the critical value, the particles fill the whole domain, but in a nonuniform way (figure 5 ).

\section{Statistical properties of the mass distribution}

\subsection{Phase-space density fluctuations and anomalous scaling}

In the previous section the Lyapunov dimension was used as an estimate to determine the presence or not of fractal clusters of particles. This dimension is not only an upper bound on the fractal dimension of the dynamical attractor; it also provides additional 


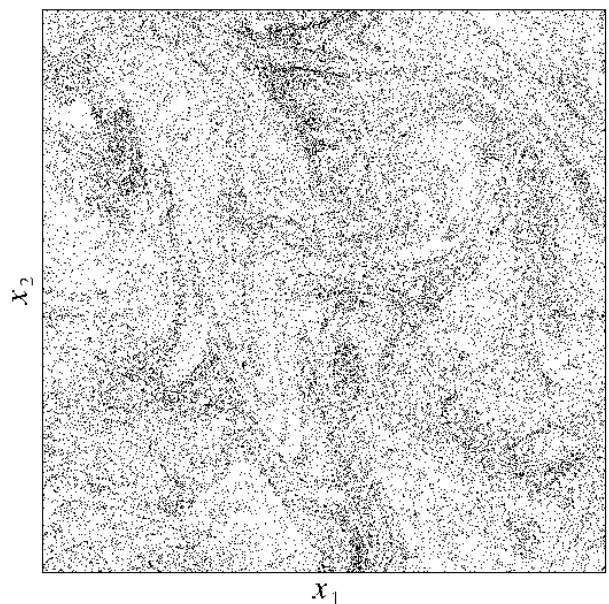

(a) $S t=10^{-3}$

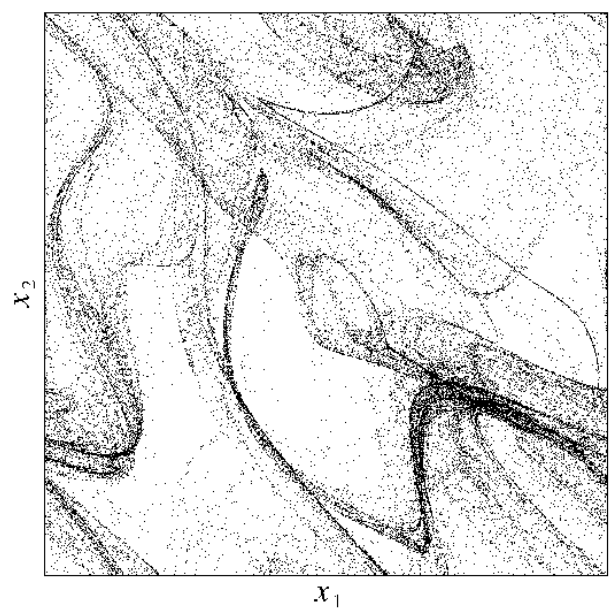

(c) $S t=10^{-1}$

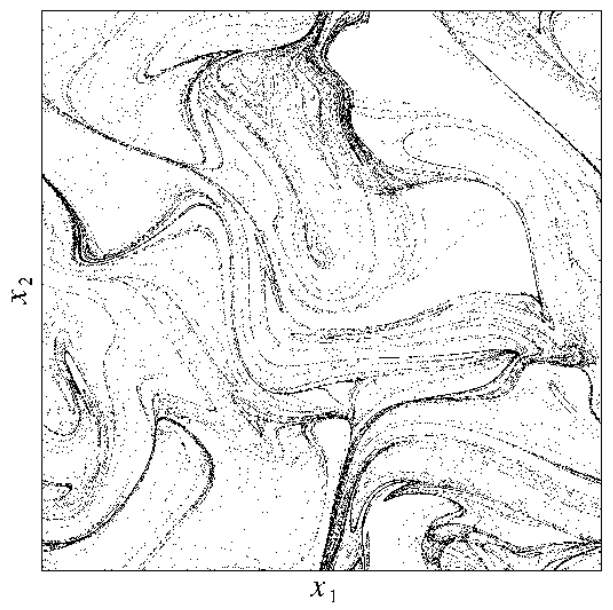

(b) $S t=10^{-2}$

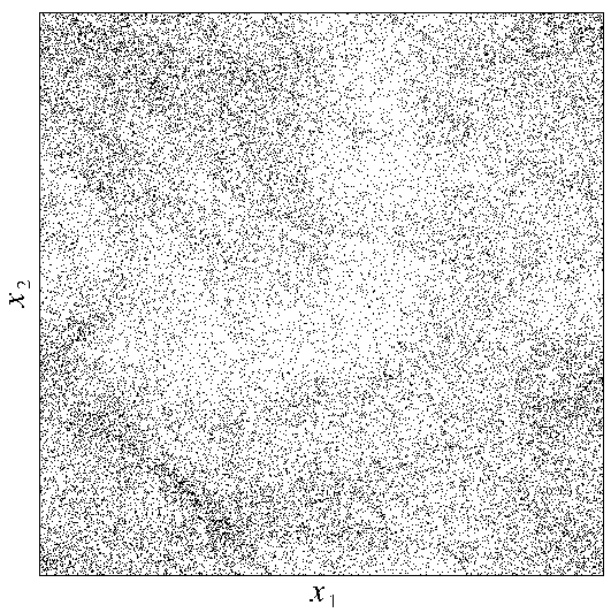

(d) $S t=1$

Figure 5. Snapshots of the positions of $N=10^{5}$ heavy particles $(\beta=0)$ associated to four different Stokes numbers as labelled. $(a),(b)$ and $(c)$ correspond to values smaller than the threshold, so that particles form fractal clusters. $(d)$ corresponds to a Stokes number larger than the critical value, so that the particles fill the whole domain.

information on the small-scale properties of the particle distribution. Consider an initial density of particles in the phase space which is uniform when projected on the position space and may or may not possess some dispersion in covelocities at each position. At a given time $t$ sufficiently large to be close to the asymptotic regime, the phase-space density field $f(\boldsymbol{x}, \boldsymbol{v}, t)$ is generally singular: its support is exactly the random dynamical attractor toward which all individual trajectories converge. This limiting density is a random equivalent of the Sinai-Ruelle-Bowen (SRB) measure, well-known in the study of dissipative dynamical systems (see, e.g., Eckmann \& Ruelle 1985; Young 2002). The density distribution along the attractor can be characterised by the mass of particles contained in small balls of radius $r$ centred on the attractor. The requirement to be on the attractor is automatically satisfied if we let the ball centre follow a given trajectory $(\mathbf{X}(t), \mathbf{V}(t))$ in phase space. Let us thus introduce the phase-space mass in a phase-space 
ball or radius $r$ around the trajectory:

$$
m_{r}(\mathbf{X}(t), \mathbf{V}(t), t) \equiv \int_{|\boldsymbol{x}|^{2}+|\boldsymbol{v}|^{2} \leqslant r^{2}} \mathrm{~d} \boldsymbol{x} \mathrm{d} \boldsymbol{v} f(\mathbf{X}(t)+\boldsymbol{x}, \mathbf{V}(t)+\boldsymbol{v}, t) .
$$

Note that $\boldsymbol{x}$ and $\boldsymbol{v}$ are here non-dimensional variables, so that it is meaningful to use the Euclidean norm in the $(\boldsymbol{x}, \boldsymbol{v})$ phase space. Under suitable non-degeneracy hypotheses for the random flow defined by the dynamics, it was shown by Ledrappier \& Young (1988) that at small scales, $m_{r}$ behaves as $r^{d_{L}}$. More precisely, for almost every particle trajectory,

$$
\frac{\ln m_{r}(\mathbf{X}(t), \mathbf{V}(t), t)}{\ln r} \rightarrow d_{L} \quad \text { as } \quad r \rightarrow 0
$$

If the limit exists then it is equal to the information dimension of the statistical-steadystate density $f$ (defined as the smallest Hausdorff dimension of a set of non-vanishing mass). In other words, the information dimension is equal to the Lyapunov dimension, identity which was conjectured by Kaplan \& Yorke and which was rigorously proved by Ledrappier \& Young for SRB measures. Of course, for a finite radius $r$ of the ball, the mass of particles does not scale exactly as $r^{d_{L}}$ but generally fluctuates. These fluctuations can be captured by investigating the scaling properties of the moments of order $n$ of the mass, which follow power laws with exponents $\xi_{n}$ at small $r$ 's:

$$
\left\langle m_{r}^{n}(\mathbf{X}(t), \mathbf{V}(t), t)\right\rangle \sim r^{\xi_{n}} \quad \text { when } \quad r \ll L .
$$

Here $\langle\cdot\rangle$ denotes averaging with respect to the realisations of the carrier flow. These exponents can be expressed in terms of the spectrum of dimensions $D_{n}$ (see Grassberger 1983; Hentschel \& Procaccia 1983) by $\xi_{n}=n D_{n+1}$. The result of Ledrappier \& Young clearly implies that $\left.\left(\mathrm{d} \xi_{n} / \mathrm{d} n\right)\right|_{n=0}=d_{L}$. There is yet another exact result which relates the exponents $\xi_{n}$ 's to the linearised dynamics. Baxendale \& Stroock (1988) showed that the exponent $\xi_{1}$ is given by the Lyapunov moments dimension associated to the generalised Lyapunov exponents (see Benzi et al. 1985). This means that it satisfies

$$
\lim _{t \rightarrow \infty} \frac{1}{t} \ln \left\langle|\boldsymbol{R}(t)|^{-\xi_{1}}\right\rangle=0,
$$

where the vector $\boldsymbol{R}(t)$ denotes the phase-space separation between two infinitesimally close trajectories. Here we observe that phenomenological arguments are proposed in Bec et al. (2003) to relate the full set of scaling exponents to the linearised system (3.1) governing the time evolution of the infinitesimal separation $\boldsymbol{R}(t)$. More precisely, for an arbitrary order $n$, the scaling exponent $\xi_{n}$ can be expressed in terms of the entropy function governing the long-time fluctuations of the stretching rates around their limiting values, the latter being precisely the Lyapunov exponents. We shall return to these matters and their implications in the conclusion (\$6).

Note that a self-similar distribution of particles would have $\xi_{n}=n d_{L}$. Because of the convexity of the function $n \mapsto \xi_{n}$ (a consequence of a Hölder's inequality applied to the moments of mass), it is clear that a necessary and sufficient condition for the exponents to depend nonlinearly on the order $n$ is that $\xi_{1}<d_{L}$. Such a nonlinear behaviour is frequently referred to as anomalous scaling since it cannot be predicted by dimensional analysis. It implies that the particle mass distribution is intermittent and in particular that its probability density function (PDF) does not have Gaussian tails. More specifically, according to the usual multifractal formalism (see, e.g., Frisch 1995), we can write the stationary PDF of the phase-space mass $m=m_{r}(\mathbf{X}(t), \mathbf{V}(t), t)$ approximately 
as

$$
p_{r}(m)=\frac{C}{m \ln r} \exp \left\{(\ln r)\left[2 d-D\left(r, \frac{\ln m}{\ln r}\right)\right]\right\} .
$$

The scaling (5.3) implies that in the limit $r \rightarrow 0$ with $(\ln m) /(\ln r)=h$ fixed, the function $D(r, h)$ tends to a limit that we denote $D_{0}(h)$. The scaling exponents $\xi_{n}$ are related to $D_{0}(h)$ by a Legendre transform

$$
\xi_{n}=2 d+\inf _{h}\left[h n-D_{0}(h)\right] .
$$

The limit $D_{0}(h)$ is frequently referred to as the multifractal spectrum of the random dynamical attractor. The names entropy function, rate function or Cramér function associated to the mass fluctuations are also sometimes used to refer to $2 d-D_{0}(h)$. The multifractal spectrum can be interpreted heuristically as the dimension of the set on which the mass in small balls scales as $r^{h}$. A straightforward consequence of the almostsure scaling of mass (5.2) is that the maximum of $D_{0}(h)$ is $2 d$ and is attained for $h=d_{L}$. Knowledge of $D_{0}(h)$ gives a very fine description of the small-scale properties of the mass distribution in phase space.

\subsection{Distribution of particle positions at low Stokes numbers}

In many applications and in particular those where interactions between inertial particles are introduced (such as collisions or chemical reactions), it is of greater interest to characterise not the phase-space mass but the position-space distribution of particles. To obtain the spatial density $\rho(\boldsymbol{x}, t)$ one must integrate the phase-space density $f$ over the covelocities. As seen in 4.2 when the dimension of the attractor is smaller than $d$, the density $\rho$ is singular with support on the projection of the attractor, that is on clusters. To investigate the distribution of particles, we consider a small ball of radius $r$ centred on a given position $\boldsymbol{x}$ which does not necessarily correspond to the position of a particle and hence may not intersect the attractor. The mass of particles contained in this ball is

$$
\bar{m}_{r}(\boldsymbol{x}, t) \equiv \int_{|\boldsymbol{y}| \leqslant \rho} \mathrm{d} \boldsymbol{y} \rho(\boldsymbol{x}+\boldsymbol{y}, t)=\int_{|\boldsymbol{y}| \leqslant \rho} \mathrm{d} \boldsymbol{y} \int \mathrm{d} \boldsymbol{v} f(\boldsymbol{x}, \boldsymbol{v}, t) .
$$

Similarly to the phase-space mass distribution, the moments of $\bar{m}_{r}$ are expected to display anomalous scaling. The usual multifractal formalism used in the previous subsection leads to defining the multifractal spectrum $\bar{D}_{0}(h)$ associated to the fluctuations of the positionspace mass $\bar{m}_{r}$. Relating the distribution of $\bar{m}_{r}$ to that of the mass in phase space $m_{r}$ requires an integration over covelocities. This can generally not be done without a better understanding of the fractal properties of the attractor in the covelocity directions. It is clear that the position variables and the covelocity variables do not play similar roles in the dynamics, so that we have a kind of anisotropy in the phase-space dynamics. This prevents us from using standard tools of the geometry of fractal sets when integrating over the covelocities.

This matter does however simplify if we limit ourselves to the asymptotics $S t \ll 1$. The position-space mass distribution can then be obtained at intermediate spatial scales from the mass distribution in the full phase space. Indeed, the covelocities of the particles associated to low Stokes numbers are within a distance order $S t$ of the phase-space manifold $\boldsymbol{v}=(1-\beta) \boldsymbol{u}(\boldsymbol{x}, t)$. Hence, when $S t \ll r / L \ll 1$, the position-space mass $\bar{m}_{r}$ is given by the particles contained in a phase-space volume also of size $r$ centred on this manifold (see figure 6). We thus introduce the exponents $\zeta_{n}$ for this intermediate asymptotic range:

$$
\left\langle\bar{m}_{r}{ }^{n}\right\rangle \sim r^{\zeta_{n}} \quad \text { for } \quad S t \ll r / L \ll 1 \text {. }
$$




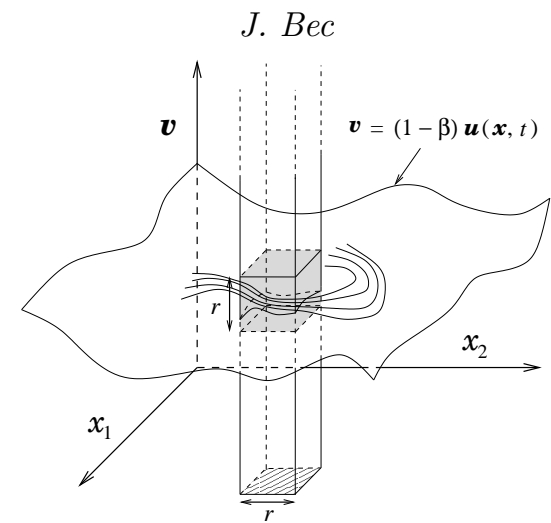

FiguRE 6. Sketch illustrating the arguments used at low Stokes number for the determination of the mass of particles $\bar{m}_{r}$ in a small volume of size $r$ in the position space. The case $d=2$ is here assumed and the two components of the covelocity in the four-dimensional phase space are schematically represented as a single variable.

We can relate the $\zeta_{n}$ 's to the exponents $\xi_{n}$ associated to the phase-space mass $m_{r}$, taking into account that $m_{r}$ is evaluated along the attractor while $\bar{m}_{r}$ is not. We have seen in 4 that for $S t \ll 1$, the particle are located on dynamical clusters whose fractal dimension is equal to that of the phase-space attractor. The probability that the ball of size $r$ intersects the fractal cluster of particles is hence $\propto r^{d-d_{H}}$. When it intersects the projection of the attractor, the mass inside the ball obviously behaves as $r^{\xi_{n}}$. We thus have

$$
\zeta_{n}=d-d_{H}+\xi_{n} .
$$

The relation (5.2) of Ledrappier \& Young between the information dimension and the Lyapunov dimension implies that $\left.\left(\mathrm{d} \zeta_{n} / \mathrm{d} n\right)\right|_{n=0}=d_{L}$. Moreover, the fact that $\xi_{0}=0$, together with relation (5.9), implies that $\zeta_{0}=d-d_{H}$. Conservation of the total mass of particles implies that the average mass in a ball of the position space scales as its volume. We hence have $\zeta_{1}=d$ and thus $\xi_{1}=d_{H}$. Note that because of the convexity of $n \mapsto \xi_{n}$, a necessary and sufficient condition to have multifractal clusters of particles (that is a non-linear dependence of the $\zeta_{n}$ ) is that $d_{H}<d_{L}$ and, of course, a value of the Stokes number below the critical clustering value.

Hunt \& Kaloshin (1997) showed that the dimension spectrum $D_{n}$ of fractal measures defined in the previous subsection is preserved under typical projections when $1<n \leqslant 2$ ("typical projections" means here almost all of them). From a direct application of this result to the steady-state phase-space density $f$, one expects that for order-unity values of the Stokes number, $\zeta_{n}=d-d_{H}+\min \left(n d, \xi_{n}\right)$ for $0<n \leqslant 1$. When $S t$ is below the critical value, it is clear from convexity of the $\xi_{n}$ 's together with $\left.\left(\mathrm{d} \xi_{n} / \mathrm{d} n\right)\right|_{n=0}=d_{L}<d$ that $\xi_{n}<n d$ for all $n$. This implies that the relation (5.9) may be extended to finite values of St. However, it is not yet completely clear whether or not this result is applicable in our setting. Indeed, because of the aforementioned anisotropy of the phase-space dynamics, it is conceivable that the projection onto the position space is not a typical one.

Our two-dimensional numerical experiments show that in the low Stokes number regime, the distribution of inertial particle positions has indeed multifractal properties. For $S t \ll 1$, particle positions almost fill the whole space and, as seen previously, their Lyapunov dimension behaves as $d_{L} \simeq d-C S t^{2}$. The scaling exponents $\zeta_{n}$ of the moments of their mass distribution are hence very close to $d n=2 n$, namely those given by simple tracers dynamics. Obtaining strong evidence for multifractal clustering requires an accurate determination of the exponents $\zeta_{n}$; this in turn requires performing long averages over the realisations of the carrier flow. Figure 7 shows the scaling exponents $\zeta_{n}$ 's 


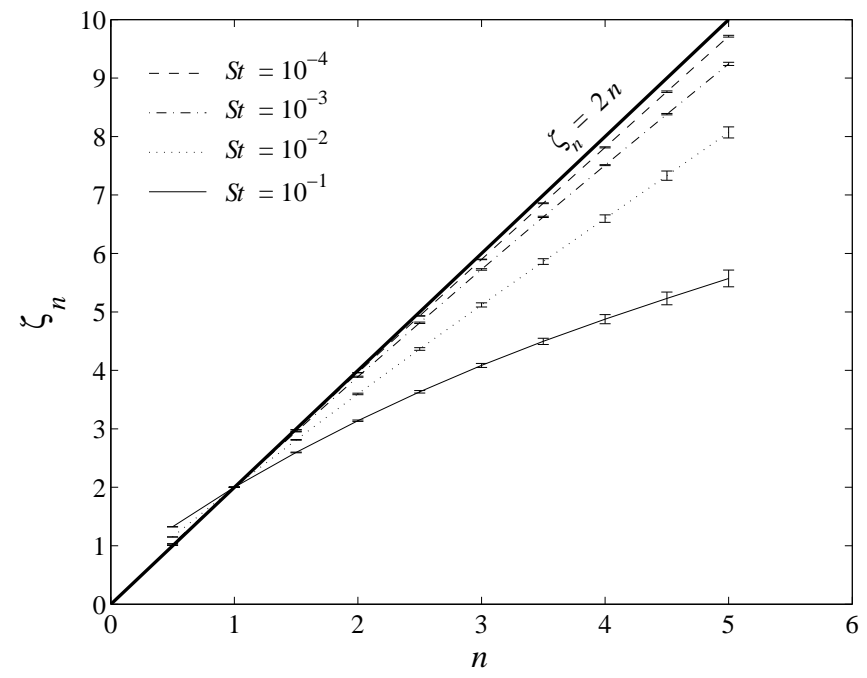

FiguRE 7. Scaling exponents $\zeta_{n}$ of the moments of the mass of particles in a small ball of radius $r$ as a function of their order $n$. The mass was calculated by box-counting with a total of $N=10^{5}$ particles and for four different values of the Stokes number, as labelled. The solid line represents the exponents associated to a uniform distribution of particles.

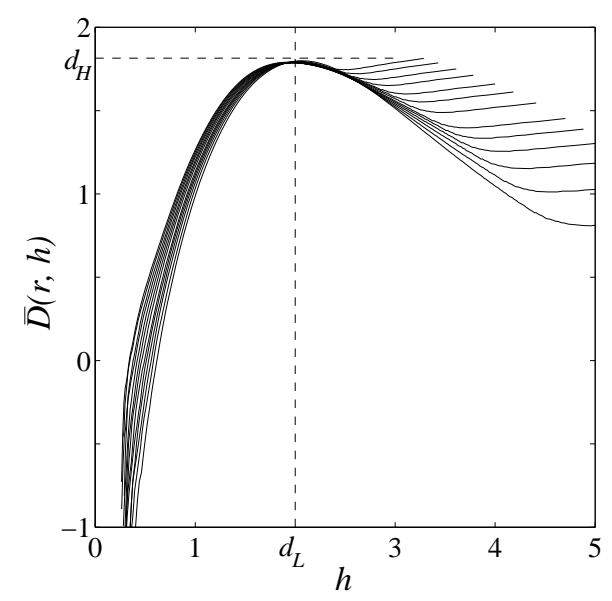

(a) $S t=10^{-3}$

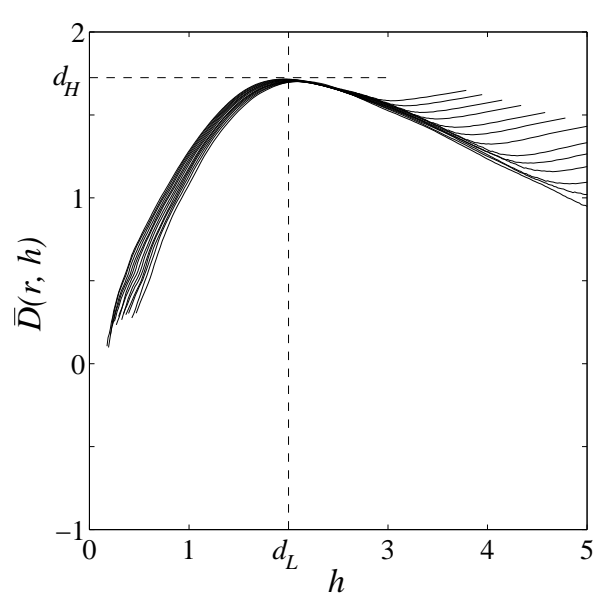

(b) $S t=10^{-2}$

Figure 8. Mass distribution for very heavy particles. The quantity $\bar{D}(r, h)$ defined by (5.10) is plotted here for different non-dimensional radii $r / L$ ranging from $5 \times 10^{-3}$ to $2 \times 10^{-1}$ and for two different values of the Stokes number as labelled. The density field is determined from the positions of $N=10^{5}$ particles. The curves approximately collapse on the function $\bar{D}_{0}(h)$ which measures mass fluctuation at those scales. It attains its maximum $d_{H}$ for $h=d_{L}$.

obtained after time averaging the mass distribution for more than $10^{4}$ turnover times of the carrier flow. Here we take very heavy particles $(\beta \ll 1)$ and plot the scaling exponents $\zeta_{n}$ for different values of the Stokes number ranging from $10^{-4}$ to $10^{-1}$. Our results show that, already at very low Stokes number, the discrepancy from a space-filling distribution is increasingly noticeable when increasing the order $n$ of the moment.

The discrepancy from a uniform distribution is even clearer when measuring the fluctuations of the position-space mass distribution. In a way similar to what was done for 
the multifractal analysis in phase space, we define

$$
\bar{D}(r, h) \equiv d-\frac{\ln p_{r}(h)}{\ln r},
$$

where $p_{r}(h)$ is the PDF of $h=\ln \bar{m}_{r} / \ln r$. Scaling of the position-space mass $\bar{m}_{r}$ in the intermediate asymptotics $S t \ll r / L \ll 1$ implies that in this range $\bar{D}(r, h)$ depends only weakly on the ball radius $r$ and becomes thus essentially a function $\bar{D}_{0}(h)$. It is easily checked that $\zeta_{n}=\inf _{h}\left(n h+d-\bar{D}_{0}(h)\right)$. Together with (5.6) and (5.9) this implies that $\overline{D_{0}}(h)=d_{H}+D_{0}(h)-2 d$. Thus, $\bar{D}_{0}(h)$ has a maximum equal to $d_{H}$ for $h=d_{L}$.

In figure 8 the functions $\bar{D}(r, h)$ corresponding to two different Stokes number are superposed for different values of the ball radius $r$ in the intermediate asymptotics. These curves were obtained in the same setting as for figure 7 namely for infinitely heavy particles $(\beta=0)$ and by time averaging over $10^{4}$ turnover times the coarse-grained density obtained from the position of $10^{5}$ particles. Pretty good scaling is observed since the curves almost collapse on a same multifractal spectrum $\bar{D}_{0}(h)$. This implies in particular that the multifractal formalism catches well the mass distribution at scales within the intermediate range $S t \ll r / L \ll 1$.

The Lyapunov dimensions associated to the two small values of $S t$ investigated here are almost equal, from which one might infer that the mass distributions are the same. In fact this is not the case: the results of the numerical experiments presented here give two kinds of evidence for a different behaviour. First, for $S t \ll 1$, the Hausdorff dimension of the attractor $d_{H}$ may not tend to $d$ as fast as $d_{L}$ (i.e. it may approach $d$ slower than quadratically). This implies that clustering may be stronger than that predicted from the analysis of the Lyapunov exponents. Second, the large fluctuations of the mass distribution (both at large and low values) which are represented by the tails of the function $\bar{D}_{0}(h)$ differ markedly for the two different low values of the Stokes numbers considered. At small Stokes numbers where the Stokes drag is very strong, the inertial particles almost follow the motion of the fluid. It is thus tempting to believe that the small effects due to particle inertia can be easily understood and quantified. As we have seen, this is not really the case: the small Stokes number asymptotic regime has a nontrivial clustering mechanism and there remains a number of open questions.

\section{Concluding remarks}

We have shown that the clustering of inertial particles in spatially smooth flow can be analysed using tools from dissipative dynamical systems. We thereby obtained evidence for the existence of a critical Stokes number below which particles form dynamical fractal clusters in position space, threshold whose existence for simple random flows was confirmed in both two and three dimensions by numerical simulations. We showed that the fluctuations in phase space of the singular mass distribution are derivable from the dimension spectrum of a random dynamical attractor. It is however difficult to "project this down" to determining mass fluctuations merely in position space, because the integration over velocities requires the knowledge of the fractal properties of the attractor in the phase-space directions associated to particle velocities. This cannot be done without a fuller understanding of the phase-space dynamics. So far, we have obtained only partial results in the asymptotics of low Stokes numbers, a regime with many practical applications.

Let us mention some further questions arising in the low Stokes number limit. The evidence is that already at very low Stokes numbers, say $10^{-4}$, particles form fractal clusters in position space. More precisely, our numerical results confirmed the prediction 
of Balkovskv et al. (2001) that the discrepancy from a uniform distribution measured from the Lyapunov dimension goes quadratically to zero as $S t \rightarrow 0$. A delicate issue is whether or not the fractal Hausdorff dimension of the clusters tends to the dimension of the ambient space that fast. Obtaining the Hausdorff dimension numerically is difficult and requires extensive computational resources. Hence, a systematic investigation of its dependence on the dynamical parameters is at the moment out of reach. There is however an alternative approach to some of the small Stokes number issues. As proposed by Maxev (1987), the particle dynamics at low Stokes numbers in an incompressible flow can be approximately described as an advection of simple passive tracers by a synthetic flow comprising a small compressible component (see also 4.2 ). In this approximation, we can then work directly in position space without having first to understand the full phase-space dynamics. In this simpler framework we can in principle take advantage of a recently proposed relation between the multifractal properties of the particle distribution (including the Hausdorff dimension) and the fluctuations of the stretching rates (Bec et al. 2003). For this we need to know the large-time statistical properties associated to the local dynamics and, in particular, the large deviations of the stretching rates from their limiting values, the Lyapunov exponents. For the moment such a determination has been made analytically only for spatially smooth compressible variants of the Kraichnan model, that is for velocity fields which are delta-correlated in time (see, e.g., Falkovich et al. 2001). The problem is that delta-correlated flow cannot be used for the low Stokes numbers regime. Indeed, the expansion requires that the Stokes numbers be much smaller than the (non-dimensional) correlation time of the carrier velocity field. A purely analytic investigation may thus be difficult, but we can use a mixed theoretical and numerical strategy for determining the multifractal properties in the low Stokes number regime: use the aforementioned relation to express them in terms of the large deviations of the stretching rates, the latter being determined numerically. This should be much easier than direct numerical determination of multifractal exponents by, for example, box-counting methods.

Finally, an important and non-trivial extension concerns the clustering properties of inertial particles at scales within the inertial range of turbulence. The dynamics of the flow at these scales is close to Kolmogorov's 1941 theory; thus the velocity is not smooth but, approximately, Hölder continuous of exponent 1/3. As a consequence, tracer particles separate in an explosive way, given by Richardson's $t^{3 / 2}$ law. It is thus important to identify and to understand the mechanisms leading to preferential concentrations of particles at those scales where there is a strong competition between clustering due to dissipative dynamics and the explosive Richardson separation. This is a challenging questions with serious methodological difficulties. On the one hand, numerical exploration of the properties of mass distribution at those scales may require very long time averages, as seen in the present study for smooth flows. Very long direct numerical simulations of three-dimensional high Reynolds number flows are hardly feasible. It may hence be necessary to focus on simpler flows, such as the synthetic turbulence of the Kraichnan model, the two-dimensional inverse cascade or large-eddy simulations. On the other hand, an analytical approach to this question can clearly not make use of standard dynamical systems tools which assume smooth dynamics. It thus seems of interest to adapt to the inertial particle dynamics models and tools which have been successfully applied in recent years to passive scalars advected by non-smooth flow in Kolmogorov-like regimes.

I am deeply grateful to U. Frisch for many interesting discussions and for his continuous support. During this work, I have benefited from stimulating and fruitful interactions with M. Cencini, K. Domelevo, G. Falkovich, P. Horvai, J. Mattingly and M. Stepanov. 
This material is based upon work supported by the European Union under contract HPRN-CT-2002-00300 and by the Indo-French Centre for the Promotion of Advanced Research (IFCPAR 2404-2). Numerical simulations were performed in the framework of the SIVAM II project at the Observatoire de la Côte d'Azur.

\section{REFERENCES}

Balkovsky, E., Falkovich, G. \& Fouxon, A. 2001 Intermittent distribution of inertial particles in turbulent flows. Phys. Rev. Lett. 86, 2790-2793.

Baxendale, P. \& Stroock, D. 1988 Large deviations and stochastic flows of diffeomorphisms. Probab. Th. Rel. Fields 80, 169-215.

BEC, J. 2003 Fractal clustering of inertial particles in random flows. Phys. Fluids 15, L81-L84.

Bec, J., Gawȩdzki, K. \& Horvai, P. 2003 Multifractal clustering in compressible flows. Preprint, http://arxiv.org/nlin.CD/0310015.

Benettin, G., Galgani, L., Giorgilli, A. \& Strelcyn, J. 1980 Lyapunov characteristic exponents for smooth dynamical systems and for hamiltonian systems; a method for computing all of them, part i and ii. Meccanica 15, 9-30.

Benzi, R., Paladin, G., Parisi, G. \& Vulpiani, A. 1985 Characterisation of intermittency in chaotic systems. J. Phys. A: Math. Gen. 18, 2157-2165.

Crisanti, A., Paladin, G. \& Vulpiani, A. 1993 Products of random matrices in statistical physics. Berlin: Springer-Verlag.

Cuzzi, J., Hogan, R., Paque, J. \& Dobrovlskis, A. 2001 Size-selective concentration of chondrules and other small particles in protoplanetary nebula turbulence. Astrophys. J. 546, 496-508.

Douady, A. \& Oesterle, J. 1980 Dimension de Hausdorff des attracteurs. C. R. Acad. Sc. Paris 24, 1135-1138.

Eckmann, J.-P. \& Ruelle, D. 1985 Ergodic theory of chaos and strange attractors. Rev. Mod. Phys. 57, 617-656.

Elperin, T., Kleeorin, N., Liberman, M., L’vov, V., Pomyalov, A. \& Rogachevskit, I. 2003 Clustering of fuel droplets and quality of spray in diesel engines. Preprint, http://arxiv.org/nlin.CD/0305017.

FAlCONeR, K. 1986 The geometry of fractal sets. Cambridge: Cambridge University Press.

Falkovich, G., Fouxon, A. \& Stepanov, M. 2002 Acceleration of rain initiation by cloud tubulence. Nature 419, 151-154.

Falkovich, G., Gawȩdzki, K. \& Vergassola, M. 2001 Particles and fields in fluid turbulence. Rev. Mod. Phys. 73, 913-976.

Frisch, U. 1995 Turbulence: the Legacy of A.N. Kolmogorov, chap. 8. Cambridge: Cambridge University Press.

Goldhirsch, I., Sulem, P.-L. \& Orszag, S. 1987 Stability and Lyapunov stability of dynamical systems: a differential approach and a numerical method. Physica D 27, 311-337.

Grassberger, P. 1983 Generalized dimensions of strange attractors. Phys. Lett. A 97, 227-230.

Hentschel, H. \& Procaccia, I. 1983 The infinite number of generalized dimensions of fractals and strange attractors. Physica D 8, 435-444.

Hunt, B. \& Kaloshin, V. 1997 How projections affect the dimension spectrum of fractal measures. Nonlinearity 10, 1031-1046.

KAPlan, J. \& YoRke, J. 1979 Functionnal differential equations and the approximation of fixed points. In Proceedings, Bonn, July 1978 (ed. H. Peitgen \& H. Walther), p. 228. Lecture Notes in Math. 730, Springer, Berlin.

Károlyi, G., PÉntek, A., Scheuring, I., Tél, T. \& Toroczkai, Z. 2000 Chaotic flow: the physics of species coexistence. Proc. Natl. Acad. Sci. USA 97, 13661.

Ledrappier, F. \& Young, L.-S. 1988 Dimension formula for random transformations. Commun. Math. Phys. 117, 529-548.

Marcus, M. \& Minc, H. 1992 A survey of matrix theory and matrix inequalities. New York: Dover Publications Inc.

MAXEY, M. 1987 The gravitational settling of aerosol particles in homogeneous turbulence and random flow fields. J. Fluid Mech. 174, 441-465. 
Maxey, M. \& Riley, J. 1983 Equation of motion of a small rigid sphere in a nonuniform flow. Phys. Fluids 26, 883-889.

Okubo, A. 1970 Horizontal dispersion of floatable particles in the vicinity of velocity singularity such as convergences. Deep-Sea Res. 17, 445-454.

Oseledets, V. 1968 A multiplicative ergodic theorem. Characteristic Lyapunov exponents of dynamical systems. Trudy Moskov. Mat. Obšč. 19, 179-210.

Pinsky, M. \& Khain, A. 1997 Turbulence effects on droplet growth and size distribution in clouds. J. Aerosol Sci. 28, 1177-1214.

SEINFELD, J. 1986 Atmospheric chemistry and physics of air pollution. New York: J. Wiley and sons.

Sigurgeirsson, H. \& Stuart, A. 2002 A model for preferential concentration. Phys. Fluids 14, 4352-4361.

Squires, K. \& Eaton, J. 1991 Preferential concentration of particles by turbulence. Phys. Fluids A 3, 1169-1178.

Squires, K. \& YAmazAKI, H. 1995 Preferential concentration of marine particles in isotropic turbulence. Deep Sea Res. Part I 42, 1989-2004.

Sundaram, S. \& Collins, L. 1997 Collision statistics in an isotropic, particle-laden turbulent suspension. i. direct numerical simulations. J. Fluid Mech. 335, 75-109.

TAYlor, G. 1921 Diffusion by continuous movements. Proc. Roy. Soc. Lond. A 20, 196-211.

Weidenschilling, S. 1995 Can gravitational instabilty form planetesimals? Icarus 116, 433435.

WEIss, J. 1991 The dynamics of enstrophy transfer in two-dimensional hydrodynamics. Physica D 48, 273-294.

Young, L.-S. 2002 What are SRB measures, and which dynamical systems have them? J. Stat. Phys. 108, 733-754. 